\title{
PULSE-STIMULATED RADIATION FROM A PLASMA AT HARMONICS OF THE GYROFREQUENCY ${ }^{\dagger}$
}

\author{
W. H. KEGEL \\ California Institute of Technology, Pasadena, California
}

(Received 29 September 1966)

\begin{abstract}
A magneto-plasma excited by two short microwave pulses at the electron gyrofrequency or a multiple thereof, which have a time separation $\tau$, is considered in the single particle approach. Due to non-linearities there arise after the second pulse, radiation maxima at the gyrofrequency and its harmonics. In a situation where the time separation of these maxima is $\tau^{*}$ (in the simplest case $\left.\tau^{*}=\tau\right)$ at the fundamental, it is $\tau^{*} / n$ at the $n$-th harmonic.

As the resonances at the harmonics are a finite Larmor radius effect, excitation at these frequencies requires a non-zero initial energy. As an example, the case is studied where the first pulse is at the gyrofrequency and the second at the second harmonic. In this case radiation maxima at $2 \omega_{0}$ occur at times $t_{l}=l \tau(l=1,2,3 \ldots)$ after the second pulse, at the fundamental, maxima arise at times $t_{l}=(2 l-1) \tau$. If there is no non-linearity involved other than the non-linear driving force, during the second pulse there arises only one radiation maximum at $t=\tau$.
\end{abstract}

\section{INTRODUCTION}

THE discussion of pulse stimulated radiation from a plasma has been restricted, up to now, essentially to the resonance at the gyrofrequency (Gould, 1965; Nonr 220 (1965); HerrmanN, 1966; Hill, 1965; Kaplan, 1966; Kegel, 1965, 1965). As the effects are non-linear, one can expect similar phenomena to occur at harmonics of the gyrofrequency. These further resonances are due to the finite ratio between Larmor radius and wavelength and are therefore weaker than the resonance at the fundamental, if the plasma is not too energetic. The resulting pulse stimulated radiation, however, might be of some interest, especially in combination with the effects at the fundamental. As the effects at different harmonics are coupled to each other, there arises the possibility of observing the stimulated radiation at a frequency which is different from that of the exciting pulses.

\section{RADIATION AT THE HARMONICS}

We consider a magneto-plasma excited by two short pulses which have a frequency $n \omega_{c}(n=1,2,3 \ldots)$ and a time separation $\tau$. We wish to calculate the radiation coming from the plasma after the second pulse.

In order to calculate the radiation we make the essential assumption that the plasma is so dilute that the single particle approach is valid. This means we neglect the interaction between the particles. For simplicity we make the further assumption that the dimensions of the plasma are small compared to the wavelength being considered.

† Research sponsored by the U.S. Office of Naval Research.

\# Work performed while the author was on leave from the Institut für Plasmaphysik, Garching bei München, Germany. 
The radiation by the plasma at one of the harmonics of the gyrofrequency depends essentially on the relative phases of the gyrating particles. The energy radiated at the $n$-th harmonic per sec into the solid angle do is given by (LANDAU and LiFSHITZ, 1953)

$$
\begin{aligned}
\mathrm{d} I_{n}= & \mathrm{d} o \frac{n^{2} e^{2} \bar{\omega}_{c}^{2}}{2 \pi c}\left\{\left|\sum_{l=1}^{N} \frac{v_{l}}{c} J_{n}\left(\frac{n v_{l}}{c} \sin \theta\right) \exp \left[\operatorname{in}\left(\omega_{c} l_{t}+\alpha_{l}\right)\right]\right|^{\mid 2}\right. \\
& +\left|\sum_{l=1}^{N} \cot \theta J_{n}\left(\frac{n w_{l}}{c} \sin \theta\right) \exp \left[i n\left(\omega_{c} l_{t}+\varkappa_{l}\right)\right]\right|^{2} \mid
\end{aligned}
$$

where $N$ is the number of particles considered, $v_{l}$ is the magnitude of the velocity of the $l$-th particle perpendicular to the magnetic field $\mathbf{H}, \omega_{c}{ }^{l}$ is its gyrofrequency, $\alpha_{l}$ its phase at $t=0$ and $\theta$ is the angle between the direction of observation and $\mathbf{H}$. If we assume the argument of the Bessel functions in (1) to be small, we have in lowest order

$$
\begin{aligned}
\mathrm{d} I_{n}= & \mathrm{d} o \frac{n^{2} e^{2} \bar{\omega}_{c}^{2}}{2 \pi c^{2 n+1}}\left(2-\sin ^{2} 0\right)\left[\frac{\sin ^{n-1} \theta}{n !}\left(\frac{n}{2}\right)^{n}\right]^{2} \\
& \times\left|\sum_{l=1}^{N} l_{l}{ }^{n} \exp \left[i n\left(\omega_{c}{ }^{l} t+\alpha_{l}\right)\right]\right|^{2} .
\end{aligned}
$$

The time variation of the radiation is determined by the last factor in (Ia). In this approximation the time variation is only due to changes in the degree of coherence of the motion of the particles. In order to study the time variation we introduce the function (Kegel)

$$
\Phi_{n}(t)=\left|\sum_{l=1}^{K} v_{l}^{n}\right|^{-2}\left|\sum_{l=1}^{j} v_{l}{ }^{n} \exp \left[i n\left(\omega_{c}{ }^{l} t+\alpha_{l}\right)\right]\right|^{\prime 2} .
$$

This function is normalized such that $0 \leqslant \Phi \leqslant 1$ and gives the radiation as a fraction of the largest possible amount for a given velocity distribution. If we introduce new quantities $t^{\prime}=t / n$ and $\alpha_{l}{ }^{\prime}=\alpha_{l} / n$ the exponential function in (2) becomes independent of $n$. This shows that one essential effect at the harmonics is that the time scale is changed due to the fact that we now deal with multipole radiation. For example, in a situation where we expect, after the second pulse, radiation peaks at the gyrofrequency having a time separation $\tau$, we expect radiation peaks at the $n$-th harmonic with a time separation $\tau / n$. As the radiation at the harmonics is multipole radiation, the weighting factor in (2) is $v^{n}$ compared to $v$ at the fundamental. As an order of magnitude, one estimates from (1a) that the intensity of the radiation peaks at the $n$-th harmonic is smaller by a factor $(\bar{v} / c)^{2 n}$ compared to the radiation at the fundamental.

The actual magnitude of the radiation peaks depends, of course, on the nonlinearities involved and the way of excitation. If the plasma is excited at one of the harmonics there is an essential non-linearity during the pulses. Besides this we restrict the discussion to the case where the relativistic mass effect causes the gyrofrequency to become energy dependent. This case was treated in detail for radiation at the fundamental in an earlier paper (KEGEL, 1967), which will be referred to as $I$.

\section{EXCITATION AT THE FUNDAMENTAL}

In this section we consider the case where the exciting pulses are at the gyrofrequency. We make the same assumptions as in $I$. We consider slight inhomogeneities 
in the magnetic field (essentially perpendicular to $\mathbf{H}$ ) by assuming a distribution $h(\eta)$ of the electrons over different gyrofrequencies, where

$$
\eta=\Delta_{i n h} \omega_{c}
$$

is the deviation from the average gyrofrequency due to differences in the magnetic field. The influence of an initial temperature is taken into account under the restriction $v_{0} \ll V$ where

$$
V=\frac{1}{2} \frac{e}{m} E_{1}{ }^{0} t_{1}
$$

$E_{1}{ }^{0}$ and $t_{1}$ being the field strength and duration of the first pulse. Under this assumption the essential effect of the initial temperature is that the particles have different energies after the first pulse. We assume a distribution $g(v)$, where $v$ is the magnitude of the velocity after the first pulse.

The velocities and relative phases of the electrons after the second pulse can be expressed as functions of $v$ and $\eta$. Taking these expressions from $I$ and replacing the sums in (2) by integrals, we find for the radiation at the second harmonic:

$$
\begin{aligned}
\Phi_{2}(t)= & \left|\iint \mathrm{d} v \mathrm{~d} \eta g(v) h(\eta) v^{* 2}\right|^{-2} \\
& \times \mid V^{2} \iint \mathrm{d} v \mathrm{~d} \eta g(v) h(\eta)\left\{D^{2} \exp (2 i f)\right. \\
& \left.+2 D(v / V) \exp \left(i f+i f_{1}\right)+(v / V)^{2} \exp \left(2 i f_{1}\right)\right\}\left.\right|^{2}
\end{aligned}
$$

with

$$
\begin{gathered}
f=\varphi_{0}+\Delta^{*} \omega_{c} t \\
f_{1}=\varphi(\tau)-\varphi_{0}+f
\end{gathered}
$$

where the asterisk indicates quantities after the second pulse, $D$ is the strength of the second pulse relative to the first one, $\varphi$ is the relative phase of the particles and $\varphi_{0}$ is the phase of the second pulse relative to a particle with $\varphi(\tau)=0$. The time $t$ is counted from the end of the second pulse. Corresponding to equation (26) of $I$, we now find

$$
\begin{aligned}
\exp (2 i f)= & \sum_{l=-\infty}^{+\infty} J_{l}(4 A t D v / V) \\
& \times \exp \left\{i \left[(2 t+l \tau)\left(\eta+A\left(1-v^{2} / V^{2}\right)\right)\right.\right. \\
& \left.\left.-(l-2) \varphi_{0}-l \pi / 2+2 A t\left(B-D^{2}-2\right)\right]\right\}
\end{aligned}
$$

where $A=\bar{\omega}_{c} V^{2} / 2 c^{2}$ and $B$ is an arbitrary constant defining the particle with respect to which $\Delta^{*} \omega_{c}$ is measured.

In the special case of an initially cold plasma, i.e. $g(v)=\delta(v-V)$, we see from (6) that there arise radiation maxima at times

Under the assumption

$$
t_{l}=-l \tau / 2 \text {. }
$$

$$
\eta_{0}+\geqslant 1
$$


where $\eta_{0}$ is a characteristic spread in the gyrofrequencies, we find for the radiation peaks

$$
\Phi_{2}\left(t_{l}\right)=\left(1+D^{2}\right)^{-2}\left\{\left|D^{2} J_{l}\left(4 A D t_{l}\right)-J_{l-2}\left(4 A D t_{2}\right)\right|^{2}+\left|2 D J_{l-1}\left(4 A D t_{l}\right)\right|^{2}\right\} .
$$

Figure 1 shows $\Phi_{2}$ for the first maximum, i.e. $l=-1$, as a function of $A \tau$, with $D=1$. The largest value is obtained for $A \tau=1 \cdot 5$. (At the fundamental the first maximum has its largest value at $A \tau=1 \cdot 1$.)

In the other limiting case of a strictly homogeneous magnetic field, i.e. $h(\eta)=\delta(\eta)$, we have the integration variable still in the Bessel functions. In order to simplify

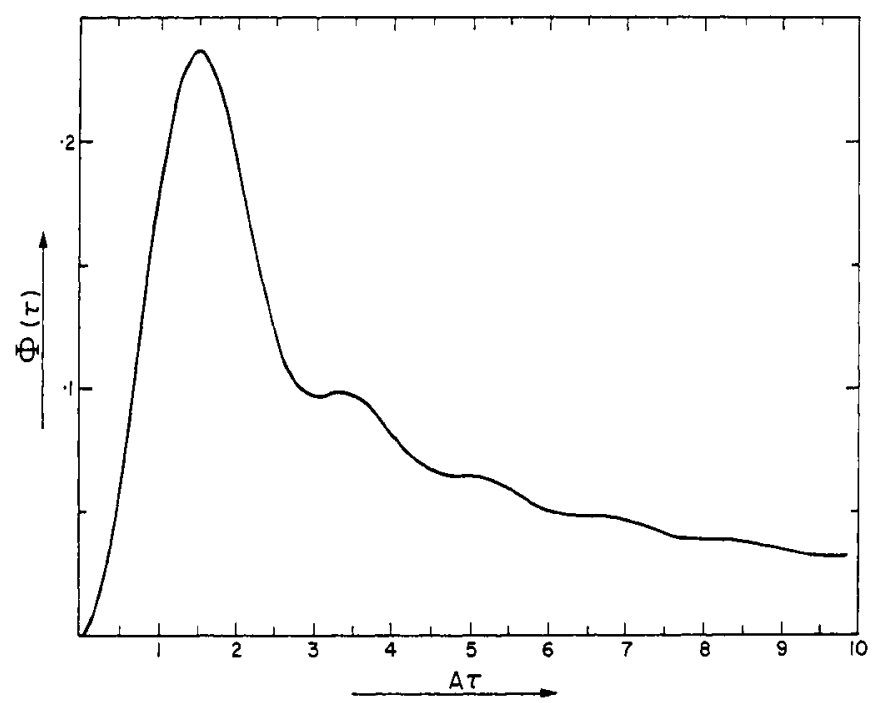

FIg. 1.-Amplitude of the first radiation maximum at the second harmonic as a function of $\tau$ in the case where the plasma is excited by two pulses at the fundamental.

the expressions we assume the argument of the Bessel functions to be large. With the same expansions as given in $I$, we then find radiation maxima at times

$$
t_{l}=-\frac{l \tau}{2(D+1)}, \quad t_{m}=\frac{m \tau}{2(D-1)} .
$$

With the assumption

where

$$
x \gg 1
$$

$$
\chi=A \tau v_{0} / V
$$

we then find for the maxima

$$
\begin{aligned}
\Phi_{2}\left(t_{l}\right) & =\frac{1}{8 \pi A D t_{l}} \cdot \frac{(1+D)^{4}}{\left(1+D^{2}\right)^{2}} \\
\Phi_{2}\left(t_{m}\right) & =\frac{1}{8 \pi A D t_{m}} \cdot \frac{(1-D)^{4}}{\left(1+D^{2}\right)^{2}}
\end{aligned}
$$


The significance of the conditions (8) and (11) is essentially the same as discussed in $I$. Especially, we find that there are virtually no radiation peaks if both conditions are simultaneously fulfilled.

\section{EXCITATION AT THE SECOND HARMONIC}

In order to obtain the resonances at the harmonics of the gyrofrequency, one has to take into account the spatial variation of the exciting field, as these resonances depend on the finite ratio of the Larmor radius to the wavelength.

We consider the influence of an electromagnetic wave on the motion of an electron in a static magnetic field $\mathbf{H}_{0}$. We choose $\mathbf{H}_{0}$ to be in the $z$-direction, the electric field $\mathbf{E}$ of the wave in the $x$-direction and its $\mathbf{k}$-vector in the $y$-direction. In this geometry the magnetic field of the wave is parallel to the static field. If we define the gyrofrequency by considering the static field only, the equations of motion (for $v \leqslant c$ ) are

$$
\begin{aligned}
& \ddot{x}=\left(-1+\frac{\dot{y}}{c}\right) \frac{e}{m} E^{0} \sin (\omega t-k y)-\omega_{c} \dot{y} \\
& \ddot{y}=\dot{x} \omega_{c}-\frac{\dot{x}}{c} \frac{e}{m} E^{0} \sin (\omega t-k y) .
\end{aligned}
$$

This system of equations for $x$ and $y$ can be uncoupled. Using the relation $\omega / k=c$, equation (14) can be integrated

$$
\dot{x}=\frac{1}{\omega} \frac{e}{m} E^{0} \cos (\omega t-k y)-\omega_{c} y+C
$$

$C$ being an integration constant. Combining (16) and (15) one obtains a second-order differential equation for $y$. We assume

$$
k y \ll 1
$$

expand the trigonometric functions in $k y$, and keep only terms linear in $k y$. Assuming further

$$
\epsilon=\frac{2}{\omega_{\mathrm{c}} c} \frac{e}{m} E^{0} \ll 1
$$

we can approximate the exact differential equation for $y$ by (see Appendix)

$$
\ddot{y}+\omega_{c}^{2} y(1-\epsilon \sin \omega t)=\frac{\omega_{c}}{\omega} \frac{e}{m} E^{0}(-1+\cos \omega t) .
$$

This is an inhomogeneous Mathieu equation. The homogeneous equation is characteristic for parametric resonance (in the present case the varied parameter is the gyrofrequency). It shows a resonance if $\omega=2 \omega_{c}$.

If one wants to study the resonances at higher multiples of the gyrofrequency, one must keep higher order terms in $k y$ when one expands the trigonometric functions.

An approximate solution of (19) can be given in terms of trigonometric functions (see Appendix). If we assume

$$
\omega=2 \omega_{c}
$$

and

$$
\frac{1}{\omega} \frac{e}{m} E^{0} \ll v(t=0) \text {. }
$$


we obtain in a co-ordinate system $\left(v_{x}{ }^{\prime}, v_{y}{ }^{\prime}\right)$ rotating with the average gyrofrequency in lowest order of $\epsilon$

$$
\begin{aligned}
& v_{x}{ }^{\prime}(t)=v_{x}{ }^{\prime}(t=0) e^{s t} \\
& v_{y}{ }^{\prime}(t)=v_{y}{ }^{\prime}(t=0) e^{-s t}
\end{aligned}
$$

with

$$
s=\frac{1}{2 c} \frac{e}{m} E^{0}=\frac{\epsilon}{4} \omega_{c}
$$

This solution is still a good approximation if the assumption (20) is substituted by

$$
\Delta \omega t_{n} \ll 1 ; \quad \frac{\Delta \omega}{\omega} \ll 1
$$

$t_{p}$ being the duration of the pulse and $\Delta(1)=\omega-2 \omega_{c}$.

We see from (22) that in lowest order there is no effect if the velocity at the onset of the pulse is zero. So we do not expect any essential effect in an experiment at the second harmonic which is similar to the experiment performed at the fundamental, i.e. if one starts with an initially cold plasma and applies two short pulses at twice the gyrofrequency.

The situation is different, however, if the first exciting pulse is at the gyrofrequency and the second pulse is at the second harmonic. In this case the first pulse provides the electrons with sufficient energy so that the second pulse can act on them.

As we obtain some interesting results under these conditions, we shall study this case in some detail. We assume the plasma to be initially cold $\left(v_{0}=0\right)$ and that some slight inhomogeneities exist in the magnetic field, essentially perpendicular to $\mathbf{H}$. The first pulse (at the gyrofrequency) gives a velocity $V$ to all particles. After the first pulse, phase differences arise due to the inhomogeneities in the magnetic field. At the onset of the second pulse the relative phase is given by

$$
\varphi(\tau)=\eta \tau
$$

where $\eta$ is given by (3) and $\tau$ is the time separation of the pulses. The second pulse (at the second harmonic) induces a quadrupole moment and we have, according to equations (22), at the end of the second pulse in the rotating frame (quantities after the second pulse are indicated by an asterisk)

with

$$
\begin{aligned}
& v_{x}{ }^{*}=V \cos [\varphi(\tau)] e^{s} \\
& v_{y}{ }^{*}=V \sin [\phi(\tau)] e^{-s}
\end{aligned}
$$

$$
S=s t_{2}
$$

where $t_{2}$ is the duration of the second pulse.

In order to calculate the relative phase $\varphi^{*}(t)$ at a time $t$ after the second pulse, we have to make an assumption about the differences in the gyrofrequencies of the different electrons. We consider two cases:

(a) The differences in gyrofrequency are given only by the inhomogeneities in the magnetic field

$$
\Delta *(1)=\eta
$$


In this case the only non-linearity taken into account is the non-linear driving force during the second pulse (KEGEL, 1966).

(b) The relativistic mass effect is essential in addition to the inhomogeneities [corresponding to $I$ equation (18)]

$$
\Delta^{*} \omega=A\left(B-v^{* 2} / V^{2}\right)+\eta
$$

At first we ask for the radiation at the gyrofrequency, i.e. we ask for $\Phi_{1}(t)$. From (2) and (26) we find

$$
\begin{aligned}
\Phi_{1}(t)= & \left(\frac{1}{2} e^{2 S}+\frac{1}{2} e^{-2 S}\right)^{-1} \cdot \mid \int \mathrm{d} \eta h(\eta) \\
& \times\left.\left\{\left[\cos [\varphi(\tau)] e^{S}+i \sin [\varphi(\tau)] e^{-S}\right] e^{i \Delta^{*} \omega t}\right\}\right|^{2} .
\end{aligned}
$$

In case (a), i.e. assuming equation (28) to be valid, we have

$$
\Phi_{1}(t) \sim\left|\frac{1}{2} \int \mathrm{d} \eta h(\eta)\left\{\left(e^{S}+e^{-S}\right) e^{i \eta(t+r)}+\left(e^{S}-e^{-S}\right) e^{-i \eta(t-\tau)}\right\}\right|^{2} .
$$

Just one radiation maximum arises at a time $t=\tau$ after the second pulse, when the second term in the curly bracket of (31) becomes independent of $\eta$. The time dependence of this maximum is given by the square of the Fourier transform of $h(\eta)$. If this is narrow compared to $\tau$, the first term does not contribute essentially and we have for the maximum

$$
\Phi_{1}(\tau)=\frac{1}{2} \frac{\left(e^{S}-e^{-S}\right)^{2}}{e^{2 S}+e^{-2 S}}
$$

if $S \ll 1$ we have

$$
\Phi_{1}(\tau) \approx S^{2}=\frac{1}{4 c^{2}}\left(\frac{e}{m} E_{2}{ }^{a} t_{2}\right)^{2} .
$$

It should be noted that at $t=0, \Phi_{1}$ is essentially zero, due to the fact that the second pulse induces a quadrupole moment but no dipole moment.

The occurrence of this single radiation maximum is due to the non-linear driving force during the second pulse and can easily be understood qualitatively by using the diagrams introduced by Gould. Figure 2 gives the distribution of the electrons in the $v^{\prime}$-space (which rotates with the average gyrofrequency) at different times; (a) at the end of the first pulse; (b) at the onset of the second pulse and (c) at the end of the second pulse. The second pulse deforms the circle in (b) into an ellipse according to equation (22). Particles at the point $E$ in this diagram change their phase during a time interval $\tau$ by $\pi \pm 2 n \pi$. So, at a time $\tau$ after the second pulse [Fig. 2(d)] the particle group $E$ coincides with group $A$ and more generally, all particles have the same phase (modulo $2 \pi$ ) which were out of phase by $\pi$ at the end of the second pulse. This shows that at this particular time a macroscopic dipole moment has been built up, the particles being distributed on a circle, the centre of which is displaced with respect to (b).

Next we consider the case (b), i.e. we assume $\Delta^{*} \omega$ as given by equation (29). From equation (26) we find

$$
v^{* 2}=V^{2}\left\{e^{-2 S}+[1+\cos 2 \varphi(\tau)] Q\right\}
$$

with

$$
Q=\frac{1}{2}\left(e^{2 S}-e^{-2 S}\right) \text {. }
$$


As we now have a cosine in the last exponential function in (30), we make an expansion into Bessel functions and obtain

$$
\begin{aligned}
\Phi_{1}(t) \sim \mid \frac{1}{2} \int \mathrm{d} & \eta h(\eta)\left\{\left(e^{S}+e^{-S}\right) \sum_{t=-\infty}^{+\infty} J_{l}(A t Q) \exp (i[\eta(t+(2 l+1) \tau)-l \pi / 2)\right. \\
& +\left.\left(e^{S}-e^{-S}\right) \sum_{m=-\infty}^{+\infty} J_{m}(A t Q) \exp (i[\eta(t+(2 m-1) \tau-m \pi / 2])\}\right|^{2} .
\end{aligned}
$$

Radiation maxima arise at times

$$
t_{l}=-(2 l+1) \tau
$$

where $l$ is an integer with the restriction $t_{l}>0$, i.e. we have radiation peaks at times $\tau, 3 \tau, 5 \tau$, etc. after the second pulse. Under the assumption (8) we find for the maxima

$$
\Phi_{1}\left(t_{l}\right)=\frac{1}{2}\left(e^{2 S}+e^{-2 S}\right)^{-1}\left\{\left.\left(e^{S}+e^{-S}\right) J_{l}\left(A t_{l} Q\right)\right|^{2}+\left|\left(e^{S}-e^{-S}\right) J_{l+1}\left(A t_{l} Q\right)\right|^{2}\right\} .
$$

If $S \ll 1$ this can be approximated by

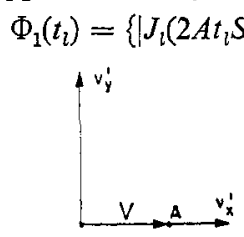

(a)

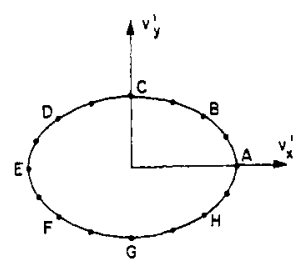

(c)
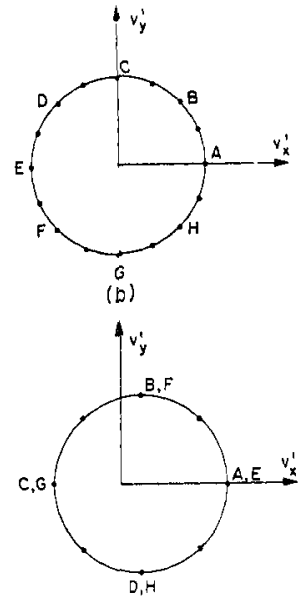

(d)

Fig. 2.-Distribution of particle velocities in a rotating velocity space; (a) at the end of the first pulse; (b) at the onset of the second pulse; (c) at the end of the second pulse; (d) at a time $\tau$ after the second pulse, the first pulse being at $\omega_{c}$ and the second at $2 \omega_{c}$.

For $l=-1$ the last term in (37) corresponds to the result (32).

Next we consider the radiation at the second harmonic, i.e. we ask for $\Phi_{2}(t)$. From (2) and (26) we find

$$
\begin{aligned}
\Phi_{2}(t)=\left(\frac{1}{2} e^{2 S}+\frac{1}{2} e^{-2 S}\right)^{-2} \mid \frac{1}{2} \int \mathrm{d} \eta h(\eta)\{2 i \sin 2 \varphi(\tau) \\
\left.+[1+\cos 2 \varphi(\tau)] e^{2 S}+[-1+\cos 2 \varphi(\tau)] e^{-2 S}\right\}\left.e^{2 \mathrm{ii \Delta} \omega^{* \omega t}}\right|^{2} .
\end{aligned}
$$

In the case (a), i.e. with equation (28) there arises again just one (weaker) maximum at a time $\tau$ after the second pulse. For this maximum we find

$$
\Phi_{2}(\tau)=\frac{\left(-2+e^{2 S}+e^{-2 S}\right)^{2}}{4\left(e^{2 S}+e^{-2 S}\right)^{2}} .
$$


If $S \ll 1$ we have

$$
\Phi_{2}(\tau)=S^{4}=\left(\frac{1}{2 c} \frac{e}{m} E_{2}{ }^{0}\right)^{4}
$$

This is essentially smaller than the maximum at the fundamental as given by (32a). In case (b), i.e. taking $\Delta^{*} \omega$ from equation (29), we find radiation maxima at times

with

$$
t=-l \tau
$$

$$
\begin{aligned}
\Phi_{2}\left(t_{l}\right)= & \left(e^{2 \mathrm{~S}}+e^{-2 \mathrm{~S}}\right)^{-2}\left\{\left|2 Q J_{l}\left(2 A t_{l} Q\right)\right|^{2}\right. \\
& \left.+\left|\left(\frac{1}{2} e^{2 S}+\frac{1}{2} e^{-2 \mathrm{~S}}-1\right)\left[J_{l-1}\left(2 A t_{l} Q\right)-J_{l+1}\left(2 A t_{l} Q\right)\right]\right|^{2}\right\}
\end{aligned}
$$

or with $S \ll 1$

$$
\Phi_{2}\left(t_{l}\right)=4 S^{2} J_{l}^{2}\left(2 A t_{l} Q\right)+S^{4}\left[J_{l-1}\left(2 A t_{l} Q\right)-J_{l+1}\left(2 A t_{l} Q\right)\right]^{2} .
$$

It should be noted that the radiation maxima have been calculated in this paper without considering any decay mechanisms. One may account for collisions by multiplying $\Phi(t)$ by a factor $\exp (-2 v t)$ where $\nu$ is a collision frequency. Besides collisions there are other effects which lead to a decay and may be important. For example, the particles considered may diffuse out of the observation region. Further, the particles can move along the lines of force due to their thermal velocities and change by this their gyrofrequency, which also leads to a decay (Gould, Nonr 220).

Acknowledgments-Fruitful discussions with R. W. Gould, K. S. H. LEE and D. MONTGOMERY are gratefully acknowledged.

\section{REFERENCES}

Bogoljubow N. N. und Mitropolski I. A. (1965) Asymptotische Methoden in der Theorie der nichtlinearen Schwingungen, Akademie.

Gould R. W. (1965) Phys. Lett. 19, 477.

Gould R. W. (1965) California Institute of Technology, Technical Report No. 28, Nonr 220 (50).

To be published.

Herrman G. F. and Whitmer R. F. (1966) Phys. Rev. 143, A122.

Hill R. M. and Kaplan D. E. (1965) Phys. Rev. Lett. 14, 1062.

KaPLAN D. E. and Hill R. M. (1966) Bull. Am. phys. Soc. 11, 538.

Kegel W. H. (1967) Plasma Phys, (J. nucl. Energy Part C) 9, 23.

KeGEL W. H. (1966) Phys. Lett. 23, 317.

Kegel W. H. and Gould R. W. (1965) Phys. Lett. 19, 531.

LANDAU L. and LIFSHITZ E. (1953) The Classical Theory of Fields, Addison-Wesley.

MEIXNER J. and SCHÄFKE F. W. (1954) Mathieusche Funktionen und Sphäroidfunktionen, Springer.

\section{APPENDIX}

\section{Solution of the Equation of Motion}

Combining equations (15) and (16) we obtain

$$
\begin{aligned}
\ddot{y}= & -\omega_{c}^{2} y+y \frac{\omega_{c}}{c} \frac{e}{m} E^{\circ} \sin (\omega t-k y)+\frac{\omega_{c}}{\omega} \frac{e}{m} E^{\circ} \cos (\omega t-k y) \\
& -\frac{1}{2} \frac{1}{\omega c}\left(\frac{e}{m} E^{\circ}\right)^{2} \sin (2 \omega t-2 k y) \\
& -\frac{C}{c} \frac{e}{m} E^{\circ} \sin (\omega t-k y)+\omega_{c} C .
\end{aligned}
$$


We now assume

$$
k y \ll 1
$$

and expand the trigonometric functions in $k y$ and neglect all terms which are quadratic in $k y$ or of higher order. Next, we determine the integration constant $C$ from the initial condition

From (A.1) we find

$$
\ddot{y}(0)+\omega_{i}^{2} y(0)=0
$$

With this we have

$$
C=-\frac{1}{\omega} \frac{e}{m} E^{\circ}
$$

$$
\begin{aligned}
\ddot{y}+\omega_{c}^{2} y= & \frac{\omega_{c}}{\omega} \frac{e}{m} E^{\circ}(-1+\cos \omega t)+\frac{1}{\omega c}\left(\frac{e}{m} E^{\circ}\right)^{2} \sin \omega t \\
& -\frac{1}{2 \omega c}\left(\frac{e}{m} E^{\circ}\right)^{2} \sin 2 \omega t \\
& +y \frac{\omega_{c}}{c} \frac{e}{m} E^{\circ}\left(2 \sin \omega t-\frac{1}{c \omega_{c}} \frac{e}{m} E^{\circ} \cos \omega t\right. \\
& \left.+\frac{1}{c \omega} \frac{e}{m} E^{\circ} \cos 2 \omega t\right) .
\end{aligned}
$$

With the assumption

$$
\varepsilon=\frac{2}{\omega_{c} c} \frac{e}{m} E^{\circ} \ll 1
$$

we can approximate (A.5) by equation (19)

$$
\ddot{y}+\omega_{c}^{2} y(1-\varepsilon \sin \omega t)=\frac{\omega_{c}}{\omega} \frac{e}{m} E^{\circ}(-1+\cos \omega t)
$$

This is an inhomogeneous Mathieu equation. One has at first to solve the homogeneous problem which is characteristic for parametric resonance and has a resonance at

$$
\omega=2 \omega_{c}
$$

If we assume $\omega$ to have the value (A.8) we have as an approximate solution of the homogeneous equation according to BOGOLJUBOW and MITROPOLSKI (1965)

with

$$
y=u \sin \omega_{c} t+v \cos \omega_{c} t+\frac{\varepsilon}{16} u \cos 3 \omega_{c} t-\frac{\varepsilon}{16} v \sin 3 \omega_{\mathrm{c}} t
$$

$$
u=C_{I} e^{s t} ; \quad v=C_{I I} e^{-s t}
$$

where $C_{l}$ and $C_{I l}$ are constants to be determined by the initial conditions and $s$ is given by (23). We have two independent solutions $y_{I}$ and $y_{I}$ with

$$
\begin{array}{ll}
u_{I}=\frac{7}{16} \varepsilon e^{s t} ; & v_{I}=e^{-s t} \\
u_{I I}=\frac{1}{\omega_{\mathrm{c}}} e^{s t} ; & v_{I I}=-\frac{\varepsilon}{16 \omega_{\mathrm{c}}} e^{-s t} .
\end{array}
$$

Using this fundamental system which solves the homogeneous equation, one finds as a solution of the inhomogeneous equation (MEIXNER, 1954)

$$
y(t)=\int_{0}^{t} f(\tau)\left[y_{I}(\tau) y_{M I}(t)-y_{n I}(\tau) y_{I}(t)\right] \mathrm{d} \tau
$$

where $f$ represents the inhomogeneous term (right-hand side) in (A.7). To this solution there must be added multiples of $y_{1}$ and $y_{I I}$ in order to fulfill the initial conditions.

Using equations (A.9-A.11) one can perform the integral in (A.12). Having found an approximate solution for $y(t)$ one can determine $x(t)$ from equation (15). If one then goes to a co-ordinate system (in velocity space) which rotates with the average gyrofrequency

$$
\begin{aligned}
& v_{x}{ }^{\prime}=-v_{x} \sin \bar{\omega}_{c} t+v_{y} \cos \bar{\omega}_{c} t \\
& v_{v}{ }^{\prime}=-v_{x} \cos \overline{\bar{\nu}}_{c} t-v_{u} \sin \bar{\omega}_{c} t
\end{aligned}
$$


and keeps only the lowest order terms (i.e. one neglects terms proportional to $\varepsilon$, $\varepsilon^{2}$, etc.) one obtains

$$
\begin{aligned}
v_{x}^{\prime}= & v_{x}^{\prime}(t=0) e^{s t}+\frac{1}{6 \omega_{0}} \frac{e}{m} E^{\circ}\left(3 \sin \omega_{c} t-\sin 3 \omega_{c} t\right) \\
v_{y}^{\prime}= & {\left[v_{y}^{\prime}(t=0)+\frac{2}{3 \omega_{c}} \frac{e}{m} E^{\circ}\right] e^{-s t} } \\
& -\frac{1}{6 \omega_{c}} \frac{e}{m} E^{\circ}\left(3 \cos \omega_{c} t+\cos 3 \omega_{c} t\right) .
\end{aligned}
$$

If one further assumes (21), i.e. that the velocity which a gyrating electron gains in one period is small compared to the initial velocity, the expression (A.14) reduces to the expression given in (22).

Equation (A.7) has further resonances in addition to that at $2 \omega_{c}$. But these show up only in approximations of higher order in $\varepsilon$ and are therefore not meaningful in the present context, as in equation (A.7) itself, higher order terms have been neglected. 